\title{
RESEARCH
}

Open Access

\section{Alterations of brain local functional connectivity in amnestic mild cognitive impairment}

Dan Zheng ${ }^{1 \dagger}$, Wei Xia ${ }^{2 \dagger}$, Zhong Quan Yi ${ }^{2}$, Pan Wen Zhao ${ }^{2}$, Jian Guo Zhong ${ }^{3}$, Hai Cun Shi ${ }^{3}$, Hua Liang Li ${ }^{3}$, Zhen Yu Dai ${ }^{4^{*}}$ and Ping Lei Pan ${ }^{2,3^{*}}$

\begin{abstract}
Background: Resting-state functional magnetic resonance imaging studies using a regional homogeneity (ReHo) method have reported that amnestic mild cognitive impairment (aMCl) was associated with abnormalities in local functional connectivity. However, their results were not conclusive.

Methods: Seed-based $d$ Mapping was used to conduct a coordinate-based meta-analysis to identify consistent ReHo alterations in aMCl.

Results: We identified 10 studies with 11 datasets suitable for inclusion, including 378 patients with aMCl and 435 healthy controls. This meta-analysis identified significant ReHo alterations in patients with aMCl relative to healthy controls, mainly within the default mode network (DMN) (bilateral posterior cingulate cortex [PCC], right angular gyrus, bilateral middle temporal gyri, and left parahippocampal gyrus/hippocampus), executive control network (right superior parietal lobule and dorsolateral prefrontal cortex), visual network (right lingual gyrus and left middle occipital gyrus), and sensorimotor network (right paracentral lobule/supplementary motor area, right postcentral gyrus and left posterior insula). Significant heterogeneity of ReHo alterations in the bilateral PCC, left parahippocampal gyrus/hippocampus, and right superior parietal lobule/angular gyrus was observed. Exploratory meta-regression analyses indicated that general cognitive function, gender distribution, age, and education level partially contributed to this heterogeneity.

Conclusions: This study provides provisional evidence that aMCl is associated with abnormal ReHo within the DMN, executive control network, visual network, and sensorimotor network. These local functional connectivity alterations suggest coexistence of functional deficits and compensation in these networks. These findings contribute to the modeling of brain functional connectomes and to a better understanding of the neural substrates of aMCl. Confounding factors merit much attention and warrant future investigations.
\end{abstract}

Keywords: Amnestic mild cognitive impairment, Default mode network, Meta-analysis, Regional homogeneity, Resting-state functional magnetic resonance imaging, Seed-based $d$ mapping

\footnotetext{
* Correspondence: daiycsy@sina.com; panpinglei@163.com

†Dan Zheng and Wei Xia contributed equally to this work.

${ }^{4}$ Department of Radiology, Affiliated Yancheng Hospital, School of Medicine,

Southeast University, West Xindu Road 2\#, Yancheng, Jiangsu Province

224001, People's Republic of China

'Department of Neurology, Affiliated Yancheng Hospital, School of Medicine,

Southeast University, West Xindu Road 2\#, Yancheng, Jiangsu Province

224001, People's Republic of China

Full list of author information is available at the end of the article
}

(c) The Author(s). 2018, corrected publication December/2018. Open Access This article is distributed under the terms of the Creative Commons Attribution 4.0 International License (http://creativecommons.org/licenses/by/4.0/), which permits

unrestricted use, distribution, and reproduction in any medium, provided you give appropriate credit to the original author(s) and the source, provide a link to the Creative Commons license, and indicate if changes were made. The Creative Commons Public Domain Dedication waiver (http://creativecommons.org/publicdomain/zero/1.0/) applies to the data made available in this article, unless otherwise stated. 


\section{Background}

Amnestic mild cognitive impairment (aMCI) is a syndrome with subjects showing memory complaints and deficits but with normal activities of daily living [1]. Individuals with aMCI are at a high risk for developing Alzheimer's dementia (AD), which is the most common type of dementia in the elderly and has been a growing public health problem worldwide [2, 3]. Understanding the neurobiology of aMCI may therefore have implications for early diagnosis and preventive interventions of $\mathrm{AD}$.

Resting-state functional magnetic resonance imaging (rs-fMRI) is a promising imaging technique to investigate regional neural activity and large-scale brain networks [4, 5]. Rs-fMRI allows for exploring complex cognitive processes in vivo without application of a task and has been extensively used to study the pathophysiology of AD and aMCI [4, 6, 7]. Regional homogeneity (ReHo) is a reliable rs-fMRI analytic algorithm to explore local functional connectivity that measures the similarity of the resting state time series between one given voxel and its neighbor voxels $[8,9]$. Over the past decade, many rs-fMRI studies have reported aberrant ReHo in patients with aMCI relative to healthy controls. ReHo can be applied to distinguish patients with aMCI from healthy controls with an accuracy of 90.32\% (sensitivity $86.21 \%$ and specificity $93.94 \%$ ) by employing a support vector machine-based approach [10]. In addition, ReHo alterations have been observed to correlate with cognitive and memory impairment in patients with aMCI [11-14]. However, despite considerable progress in the better understanding of aMCI pathophysiology, the findings from ReHo studies have been less consistent than expected. For example, ReHo alterations in the posterior cingulate cortex (PCC)/precuneus in patients with aMCI relative to healthy controls have been controversial. Decreased ReHo, increased ReHo, or null findings in this region have been reported. Thus, it is of keen interest to overcome such inconsistency and characterize ReHo alterations in patients with aMCI by quantitatively pooling these studies. However, no such study has so far been conducted.

Our primary objective of the present study was to identify the consistent ReHo alterations in aMCI across studies via a meta-analytic approach. Seed-based $d$ Mapping (SDM), a fully-validated technique for coordinate-based meta-analysis of neuroimaging studies [15-17], was utilized to quantitatively synthesis the whole-brain ReHo results in a voxel-wise manner. Additionally, we aimed to conduct meta-regression analyses to explore the potential impact of relevant demographic and clinical variables on $\mathrm{ReHo}$ changes in patients with aMCI.

\section{Methods}

\section{Literature search and study selection}

The electronic databases of PubMed, Web of Science, and Embase were systematically searched up until 14 July, 2017, using the following keywords and combinations: ("mild cognitive impairment" or "mci") AND ("regional homogeneity" or "ReHo" or "local connectivity"). Reference lists from relevant studies were further reviewed to detect additional suitable articles. The search was updated on 8 May, 2018.

Study selection was according to the following inclusion criteria: 1) Patients enrollment met the diagnostic criteria for aMCI; 2) the study employed rs-fMRI to measure ReHo differences between patients with aMCI and matched healthy controls; 3 ) the study utilized a voxel-based statistical analysis at the whole-brain level; 4) three-dimensional stereotactic coordinates in either Talairach or Montreal Neurological Institute (MNI) space were explicitly reported; and 5) the study was an original article that was peer-reviewed and published in an English-language journal. Studies were excluded if they enrolled patients with non-aMCI. Studies were also excluded if they limited their analyses to specific regions of interest (ROIs), or used two-dimensional analyses, or did not report peak stereotactic coordinates. Only one study was selected in the final meta-analysis in case that two or more published studies had the same first author and data acquisition methods, and similar patient demographic characteristics, clinical variables, data analysis, and imaging results. Only pre-treatment or baseline data were included in case of longitudinal studies.

We used a 20-point checklist (Additional file 1), which was based on previous neuroimaging meta-analyses to evaluate the quality of each eligible study. Our study was performed in accordance with the Meta-analysis of Observational Studies in Epidemiology (MOOSE) guidelines [18].

\section{Data analysis}

Main voxel-wise meta-analysis by pooling all included studies The current version 5.15 of the SDM software package (www.sdmproject.com) was used to test for significant spatial convergence of ReHo findings across studies in patients with aMCI relative to healthy controls. The SDM approaches have been widely applied in neuropsychiatric disorders and the processes have been described in detail elsewhere [15, 19-25]. Peak three-dimensional stereotactic coordinates and their effect sizes of ReHo differences between patients with aMCI and healthy controls were firstly extracted from each study [19, 22]. A standard MNI map of ReHo changes for each study was then separately recreated with an un-normalised anisotropic Gaussian kernel (full width at half maximum $[$ FWHM] $=20 \mathrm{~mm})[19,22,23]$. Of note, this 
Gaussian kernel is not used to smooth any image but to assign indicators of proximity to reported coordinates [20]. The mean map was further generated by voxel-wise calculation of the mean of the study maps. A random-effect model was applied, which takes into account the sample size, intra-study variability, and between-study heterogeneity $[19,22,23]$. The following thresholds were used to obtain the final SDM statistical map: uncorrected $p<0.005$, peak height $\mathrm{Z} \geq$ 1 , and cluster extent $\geq 20$ voxels. It should be noted that this uncorrected significance has been found in SDM method to be approximately equivalent to a corrected $p$-value $=0.05[19,22,23]$.

\section{Reliability analysis}

To test the reliability of the brain areas identified in the main voxel-wise meta-analysis, we performed whole-brain voxel-based jackknife sensitivity analyses by repeating the same analyses with the consecutive exclusion of each study $[19,20,23,24]$.

\section{Heterogeneity analysis}

For assessing between-study heterogeneity of individual clusters, a random-effect model with $\mathrm{Q}$ statistics was utilized. $P<0.005$, peak height $\mathrm{z} \geq 1$, and cluster extent $\geq 20$ voxels were considered significant [26].

\section{Publication bias analysis}

Possible publication bias was assessed with an Egger test by extracting the values from the relevant peaks in the main voxel-wise meta-analysis. A threshold at $p<0.05$ indicated statistical significance.

\section{Subgroup meta-analysis}

Subgroup meta-analyses of studies using 3.0 T MRI scanners $(N=9)$ and of studies that matched for sex between patients with aMCI and healthy controls $(N=10)$ were conducted to investigate the possible effects of the results on the overall conclusions.

\section{Meta-regression analysis}

A meta-regression analysis was conducted to assess the severity of general cognitive function impairment examined by MMSE scores that correlated with the ReHo alterations in the patient samples. We also investigated the influence of demographic factors, such as gender distribution (female ratio), age, and educational level, on the ReHo changes in the aMCI samples. These analyses were performed with simple linear regression models. A stringent statistical threshold at $p<0.0005$ and a cluster extent of 20 voxels were used [19, 21].

\section{Results}

Literature search and study selection

The systematic literature search yielded 305 titles and abstracts. Of these results, we identified 10 studies with 11 datasets suitable for final inclusion (378 patients, 435 controls) [10, 12-14, 27-32]. The detailed study selection process for the meta-analysis is shown in the flowchart Fig. 1. No significant differences were observed between the aMCI samples and $\mathrm{HC}$ groups regarding mean age (standardized mean difference $[\mathrm{SMD}]=0.061$; $95 \%$ confidence interval $[\mathrm{CI}]=-0.096$ to $0.218, \mathrm{z}=0.76$, $p=0.45)$ or education level $(\mathrm{SMD}=-0.26 ; 95 \% \mathrm{CI}=-$ 0.57 to $0.037, \mathrm{z}=1.72, p=0.086$ ). However, an unbalance of gender distribution between the aMCI samples and $\mathrm{HC}$ groups (relative risk $=1.22,95 \% \mathrm{CI}=1.05$ to 1.40 , $\mathrm{z}=2.69, \quad p=0.007)$ was observed. All the studies included except for the study by Cha [32] matched for sex. In addition, the aMCI groups had significantly lower mean MMSE scores than that of the HC groups $(\mathrm{SMD}=-1.70 ; 95 \% \mathrm{CI}=-2.26$ to $-1.15, \mathrm{z}=$ $6.00, p=0.000)$. The quality of each included study was acceptable, with the quality score no less than 18 (a maximum score $=20$ ). The demographic, clinical, and technical characteristics as well as the quality score of each eligible study are summarized in Table 1.

ReHo differences between patients with aMCI and healthy controls

The voxel-wise SDM results showed increased ReHo in the left parahippocampal gyrus (extending to the left hippocampus), right lingual gyrus (extending to the left middle occipital gyrus), right paracentral lobule (extending to the supplementary motor area), and postcentral gyrus, and decreased ReHo in the left insula, right superior parietal lobule (extending to the right angular gyrus), right dorsolateral prefrontal cortex (DLPFC), bilateral PCC, and bilateral middle temporal gyri in patients with aMCI compared to healthy controls. Details regarding the location, size, and peak density of each cluster are presented in Table 2. The SDM map is illustrated in Fig. 2.

\section{Reliability analysis}

The sensitivity analyses revealed that most of the areas found in the SDM map had very high reliability, with areas replicable in at least 9 out of 11 combinations of datasets. Decreased ReHo in the bilateral PCC was less robust than other areas, replicable in 7 out of 11 combinations of datasets (Table 2).

\section{Heterogeneity analysis}

Analysis of heterogeneity revealed that brain areas with altered $\mathrm{ReHo}$ in the bilateral PCC/precuneus, left 


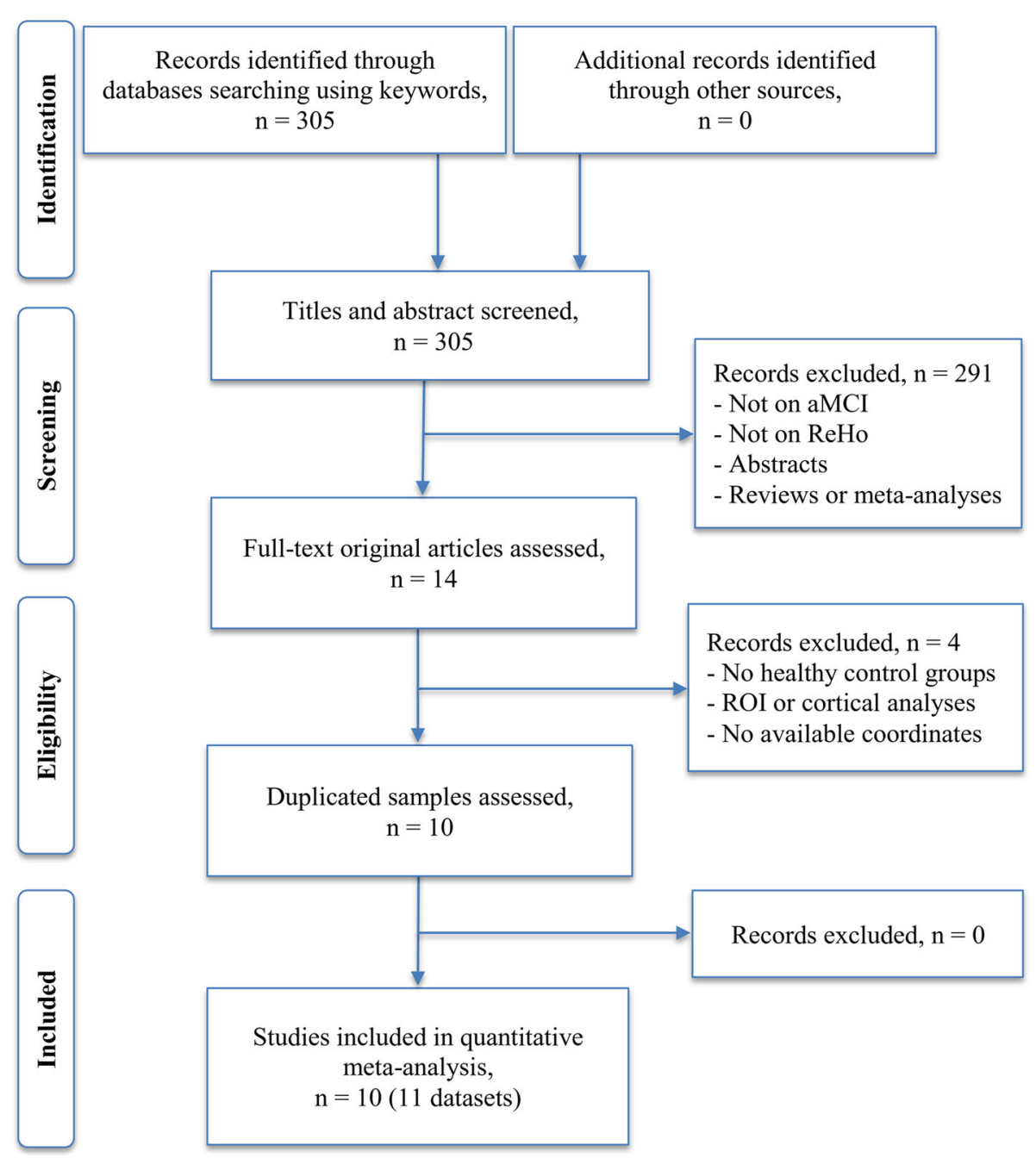

Fig. 1 Flow chart for the literature selection. Abbreviations: aMCl, amnestic mild cognitive impairment; ReHo, regional homogeneity; ROI, region of interest

supplementary motor area, right superior parietal lobule (extending to the angular gyrus), left fusiform gyrus, and left lingual gyrus had significant betweenstudy heterogeneity. The results from the heterogeneity analysis are summarized in Table 3 and Fig. 3.

\section{Publication bias analysis}

Publication biases were detected in the bilateral PCC $(p=0.011)$ and left parahippocampal gyrus (extending to the left hippocampus) $(p=0.034)$. No publication biases for other brain regions identified in the SDM map were observed, which were revealed by the non-significant Egger's tests (Table 2).

\section{Subgroup meta-analysis}

The subgroup meta-analysis of studies using 3.0 T MRI scanners $(N=9)$ demonstrated that patients with aMCI compared to healthy controls exhibited increased ReHo in the bilateral lingual gyri (extending to the left middle occipital gyrus and calcarine fissure/surrounding cortex), left parahippocampal gyrus (extending to the left hippocampus), right paracentral lobule (extending to the right supplementary motor area), right postcentral gyrus, and right lingual gyrus, and decreased $\mathrm{ReHo}$ in the right angular gyrus (extending to the right superior parietal lobule), left fusiform gyrus (extending to left the inferior temporal gyrus), right DLPFC, left insula, and left PCC (extending to the left precuneus). The jackknife sensitivity analyses revealed that regions with ReHo alterations in the right postcentral gyrus, and right lingual gyrus, and left PCC (extending to the left precuneus) were less robust as they were only replicable in 4,5 , and 6 out of 9 combinations of datasets, respectively. Other regions showed relative robustness as they were replicable in at least 7 out of 9 combinations of datasets. The heterogeneity analysis showed significant statistical heterogeneity 


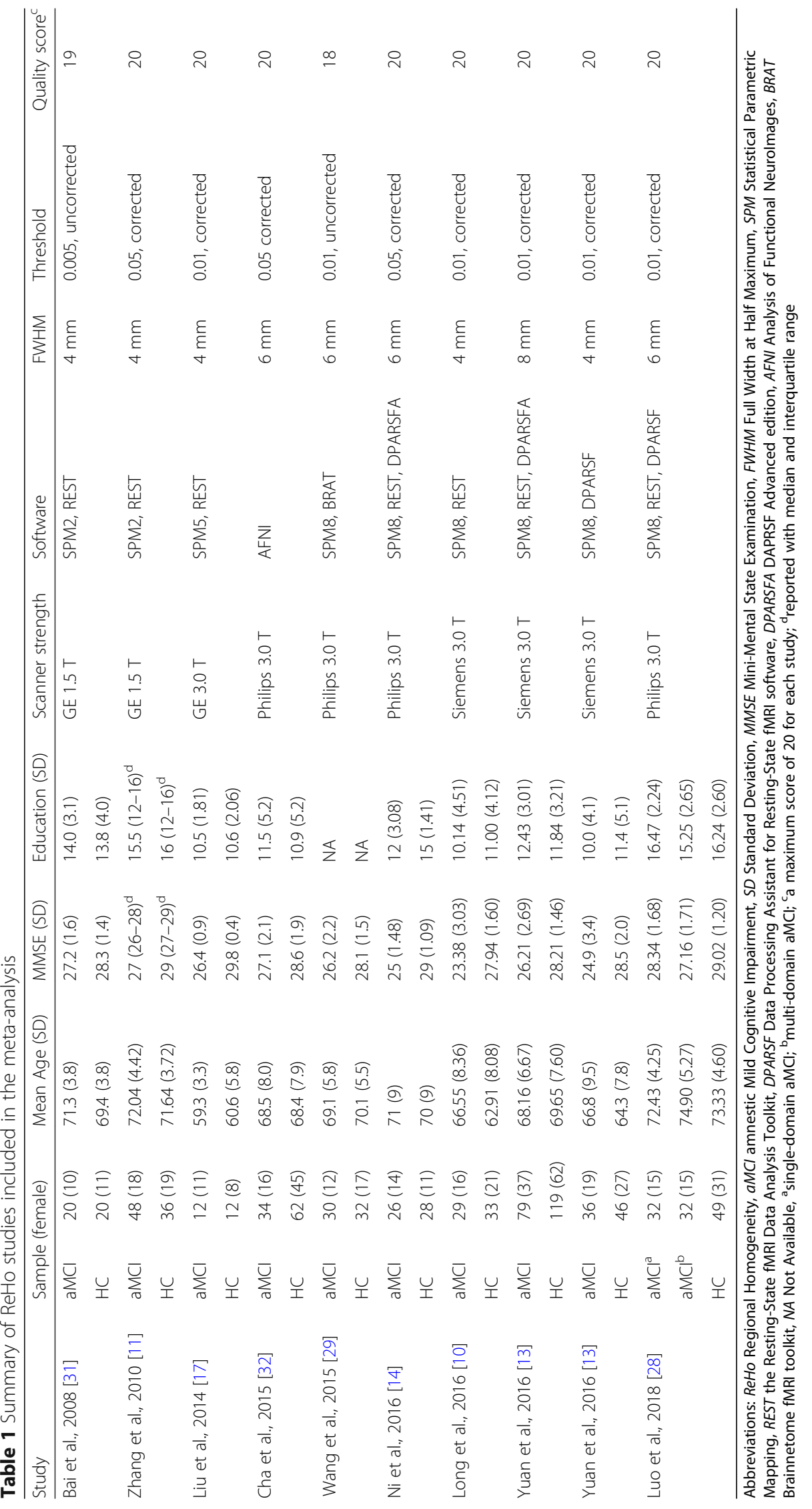


Table 2 ReHo alterations in patients with aMCI relative to healthy controls

\begin{tabular}{|c|c|c|c|c|c|c|c|}
\hline & Anatomical label & $\begin{array}{l}\text { Peak MNI coordinate } \\
(x, y, z)\end{array}$ & Voxels & SDM-Z value & $\begin{array}{l}p \text { value } \\
\text { (SDM) }\end{array}$ & $\begin{array}{l}\text { Sensitivity } \\
\text { analysis }\end{array}$ & $\begin{array}{l}p \text { value } \\
\text { (Egger's test) }\end{array}$ \\
\hline \multirow[t]{5}{*}{ Increased ReHo } & $\begin{array}{l}\text { A. Left parahippocampal gyrus/ } \\
\text { hippocampus (BAs } 28 \text { and 35) }\end{array}$ & $-22,-20,-22$ & 197 & 1.5 & 0.0003 & $10 / 11$ & 0.03 \\
\hline & $\begin{array}{l}\text { B. Right lingual gyrus/left middle } \\
\text { occipital gyrus (BAs } 17 \text { and 18) }\end{array}$ & $10,-88,-6$ & 488 & 1.4 & 0.0005 & $10 / 11$ & 0.8 \\
\hline & $\begin{array}{l}\text { C. Right paracentral lobule/ } \\
\text { supplementary motor area } \\
\text { (BAs } 6 \text { and 4) }\end{array}$ & $10,-30,54$ & 190 & 1.5 & 0.0003 & $9 / 11$ & 0.6 \\
\hline & D. Right postcentral gyrus (BA 3) & $34,-28,44$ & 45 & 1.3 & 0.001 & $9 / 11$ & 0.8 \\
\hline & E. Right lingual gyrus (BA 18) G & $10,-66,-6$ & 38 & 1.0 & 0.004 & $9 / 11$ & 0.9 \\
\hline \multirow[t]{6}{*}{ Decreased ReHo } & F. Left insula (BA13) & $-38,-10,8$ & 525 & -1.8 & 0.001 & $9 / 11$ & 0.9 \\
\hline & $\begin{array}{l}\text { G. Right superior parietal lobule/ } \\
\text { angular gyrus (BAs } 7 \text { and 39) }\end{array}$ & $34,-72,42$ & 306 & -1.9 & 0.0007 & $11 / 11$ & 0.9 \\
\hline & $\begin{array}{l}\text { H. Right dorsolateral prefrontal } \\
\text { cortex (BA 9) }\end{array}$ & $34,10,36$ & 94 & -1.9 & 0.0005 & $9 / 11$ & 0.2 \\
\hline & $\begin{array}{l}\text { I. Right/Left posterior cingulate } \\
\text { gyrus (BAs } 23 \text { and 30) }\end{array}$ & $2,-48,24$ & 67 & -1.7 & 0.002 & $7 / 11$ & 0.01 \\
\hline & J. Left middle temporal gyrus (BA 37) & $-50,-70,0$ & 22 & -1.6 & 0.003 & $9 / 11$ & 1.0 \\
\hline & $\begin{array}{l}\text { K. Right middle temporal gyrus } \\
\text { (BA 21) }\end{array}$ & $54,2,-20$ & 16 & -1.6 & 0.004 & $9 / 11$ & 0.9 \\
\hline
\end{tabular}

Abbreviations: ReHo Regional Homogeneity, aMCl amnestic Mild Cognitive Impairment, MNI Montreal Neurological Institute, SDM Seed-based $d$ Mapping, BA Brodmann area

of ReHo in the right angular gyrus (extending to the right superior parietal lobule) and left PCC (extending to the left precuneus). No significant statistical heterogeneity of ReHo in other regions reported above was observed. Egger's tests revealed no publication biases in the regions reported (all $p>0.05$ ). The detailed results are presented in Additional file 2.

The subgroup meta-analysis of studies that matched for sex between patients with aMCI and healthy controls $(N=10)$ showed that patients with aMCI relative to healthy controls exhibited increased ReHo in the right paracentral lobule (extending to the supplementary motor area and postcentral gyrus), right lingual gyrus (extending to the bilateral calcarine fissure/surrounding cortex), and left parahippocampal gyrus (extending to the left hippocampus), and decreased ReHo in the bilateral PCC (extending to the precuneus), right middle temporal gyrus, right DLPFC, and right angular gyrus (extending to the right inferior parietal lobule). The jackknife sensitivity analyses revealed that regions with ReHo alterations in the in the right paracentral lobule (extending to the supplementary motor area and postcentral gyrus), right lingual gyrus (extending to the bilateral calcarine fissure/surrounding cortex), left parahippocampal gyrus (extending to the left hippocampus), bilateral PCC (extending to the precuneus), and right angular gyrus (extending to the right inferior parietal lobule) were replicable in 9 combinations out of 10 datasets. Right middle temporal gyrus and right dorsolateral prefrontal cortex (BA 9) were replicable in 7 and 8 combinations out of 10 datasets, respectively. The heterogeneity analysis showed significant statistical heterogeneity of ReHo in the left parahippocampal gyrus (extending to the left hippocampus), bilateral PCC (extending to the precuneus), and right angular gyrus (extending to the right inferior parietal lobule). No significant statistical heterogeneity of ReHo in other regions reported above was observed. Egger's tests revealed publication biases in the right lingual gyrus (extending to the bilateral calcarine fissure/surrounding cortex) and bilateral PCC (extending to the precuneus) $(p<0.05)$. The detailed results are presented in Additional file 3.

\section{Meta-regression analysis}

The meta-regression analysis showed that severer general cognitive function impairment revealed by lower MMSE score in the aMCI samples was associated with less $\mathrm{ReHo}$ in the right angular gyrus/supramarginal gyrus (Fig. 4a), right DLPFC (Fig. 4b), and left inferior temporal gyrus (Fig. 4c). More females in the aMCI samples (female ratio) were related to less $\mathrm{ReHo}$ in the left PCC/precuneus (Fig. 5a) and greater ReHo in the left pre/postcentral gyrus (Fig. 5b). Mean age in the patient samples was negatively correlated with $\mathrm{ReHo}$ in the right precuneus (Fig. 6). Higher educational level in the aMCI sample was associated with lower ReHo in the left calcarine fissure/surrounding cortex (Fig. 7a) and higher ReHo in left fusiform gyrus/inferior temporal gyrus (Fig. 7b) and right angular gyrus/superior temporal gyrus (Fig. 7c). 


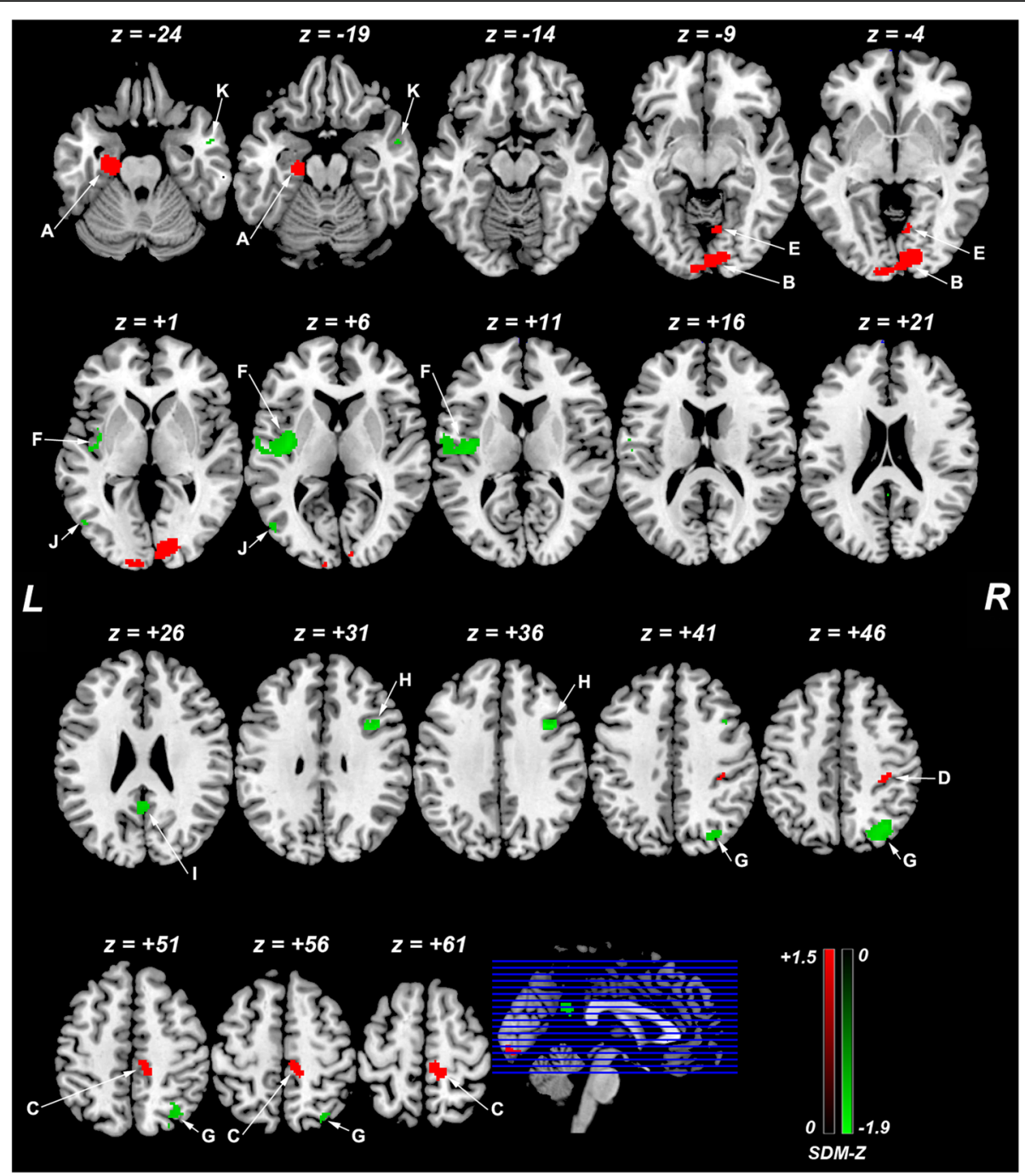

Fig. 2 ReHo differences in patients with aMCl and healthy controls from the main voxel-wise meta-analysis. Abbreviations: ReHo, regional homogeneity; aMCl, amnestic mild cognitive impairment; L, left; R, right; SDM, Seed-based d Mapping; a, left parahippocampal gyrus (extending to the left hippocampus); $\mathbf{b}$, right lingual gyrus (extending to the left middle occipital gyrus); $\mathbf{c}$, right paracentral lobule (extending to the supplementary motor area); $\mathbf{d}$, postcentral gyrus; $\mathbf{e}$, right lingual gyrus; $\mathbf{f}$, left insula, $\mathbf{g}$, right superior parietal lobule (extending to the right angular gyrus); $\mathbf{h}$, right dorsolateral prefrontal cortex; $\mathbf{i}$, bilateral posterior cingulate cortex, $\mathbf{j}$, left middle temporal gyrus, $\mathbf{k}$, right left middle temporal gyrus. The color bar (increases of ReHo in red and decreases in green) indicates the maximum and the minimum SDM-Z values

Table 3 Regions of ReHo heterogeneity from the SDM analysis

\begin{tabular}{|c|c|c|c|c|}
\hline Anatomical regions & Maximum MNI coordinate $(x, y, z)$ & Voxels & SDM-Z value & $p$ value \\
\hline A. Right/Left posterior cingulate gyrus/precuneus (BA 23) & $-8,-50,38$ & 919 & 4.0 & 0.0001 \\
\hline B. Left supplementary motor area (BA 6) & $-2,-6,52$ & 113 & 3.4 & 0.0008 \\
\hline C. Right superior parietal lobule/angular gyrus (BA7) & $34,-72,52$ & 53 & 3.3 & 0.001 \\
\hline D. Left fusiform gyrus (BA 19) & $-40,-64,-18$ & 54 & 3.2 & 0.001 \\
\hline E. Left lingual gyrus (BA 17) & $0,-94,0$ & 17 & 2.8 & 0.003 \\
\hline
\end{tabular}




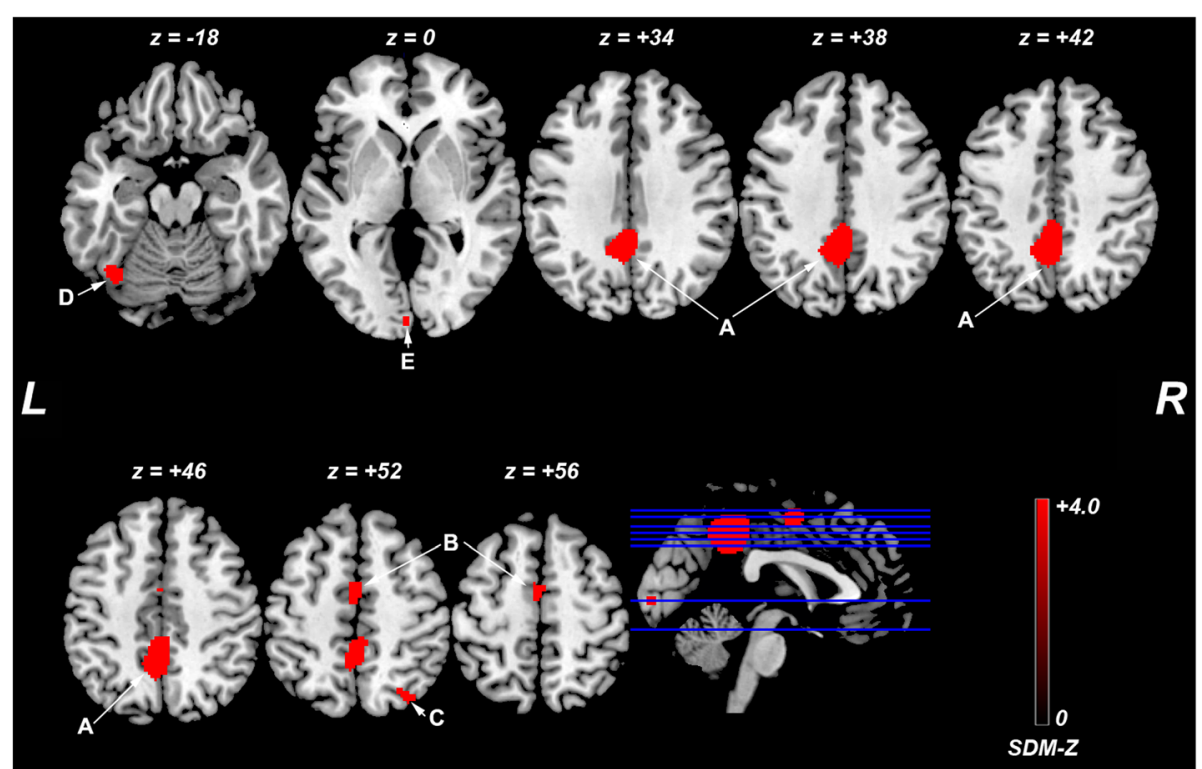

Fig. 3 Regions with ReHo heterogeneity across studies. Abbreviations: SDM, Seed-based $d$ Mapping; L, left; R, right; a, bilateral posterior cingulate cortex (extending to the precuneus); $\mathbf{b}$, left supplementary motor area, $\mathbf{c}$, right superior parietal lobule (extending to the angular gyrus); $\mathbf{d}$, left fusiform gyrus, e, left lingual gyrus. The color bar indicates the maximum and the minimum SDM-Z values

Table 4 summarizes the results of the meta-regression analyses.

\section{Discussion}

To the best of our knowledge, this is the first study to employ a meta-analytic approach to demonstrate ReHo differences between patients with aMCI and healthy controls. This meta-analysis identified significant brain ReHo alterations in patients with aMCI relative to healthy controls, mainly within the default mode network (DMN) (bilateral PCC, right angular gyrus, bilateral middle temporal gyri, and left parahippocampal gyrus/hippocampus), executive control network (right superior parietal lobule and DLPFC), visual network (right lingual gyrus and left middle occipital gyrus), and sensorimotor network (right paracentral lobule/supplementary motor area, right postcentral gyrus and left posterior insula). It should be noted that significant heterogeneity of ReHo alterations in the bilateral PCC, left parahippocampal gyrus/hippocampus, and right superior parietal lobule/angular gyrus was observed in the meta-analysis. The subgroup meta-analyses revealed that MR field-strength and sex partly affected the overall results. Further meta-regression analyses indicated that some confounding factors, such as general cognitive function, gender distribution, age, and education level could partially contribute to the heterogeneity across studies.

This voxel-wise meta-analysis identified significant decreases of ReHo in the bilateral PCC, right angular gyrus, and bilateral middle temporal gyri and increases of ReHo in the left parahippocampal gyrus/hippocampus in patients with aMCI relative to healthy controls. These regions of ReHo alterations are critical hubs of the DMN [33]. The DMN, which is implicated in self-referential and memory processing, is the most studied brain cognitive network in aMCI and $\mathrm{AD}$ because it is preferentially susceptible to neurodegeneration and involves early in the AD pathophysiology $[33,34]$. Convergent evidence suggests that remote functional connectivity in the DMN in AD and aMCI is reorganized, which correlates with cognitive impairments [33-35]. Our findings in alterations of local functional connectivity within the DMN yielded complimentary insights, which contribute to the modeling of brain functional connectomes of aMCI. Functional connectivity alterations in the DMN have been reported as a predictor of conversion from aMCI to AD [36, 37]. Even more importantly, the DMN has been gaining attention as a potential target of non-invasive brain stimulation for $\mathrm{AD}$ [33]. Both decreases and increases of ReHo were found in the DMN. This coexistence of functional deficits and compensation in aMCI was also suggested by previous studies [38-43]. The parahippocampal gyrus and hippocampus located in the medial temporal lobe, where the hallmark of pathological changes in $\mathrm{AD}$, are critical structures responsible for mnestic functions. Increased ReHo in the parahippocampal gyrus/ hippocampus observed in this meta-analysis may serve to compensate for its neurodegeneration underlying memory impairments in patients with aMCI. It 


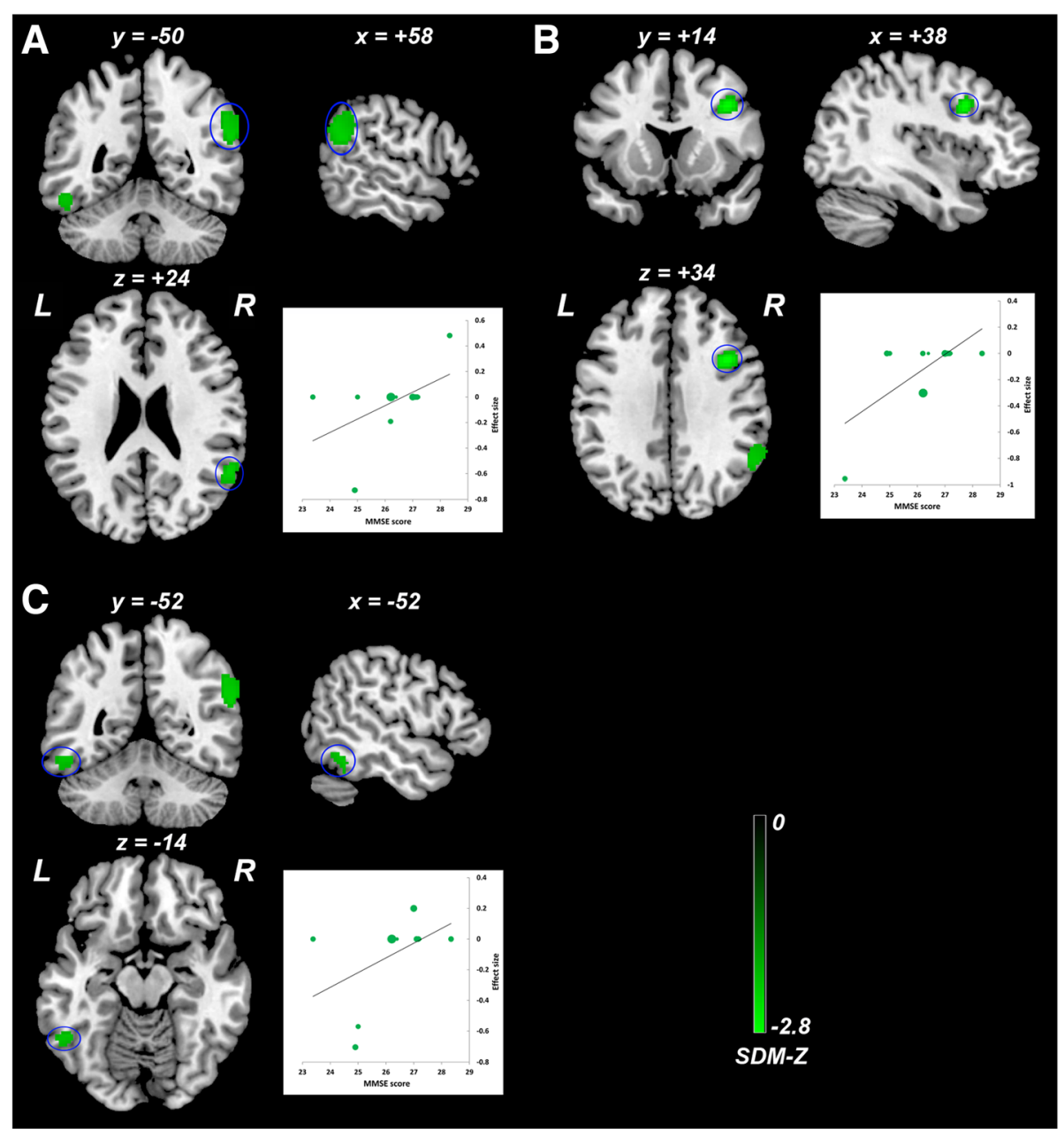

Fig. 4 Meta-regression analysis of ReHo alterations against the mean MMSE scores across studies. Lower mean MMSE score in the aMCI sample was associated with lower ReHo in the right angular gyrus/supramarginal gyrus (a), right DLPFC (b), and left inferior temporal gyrus (c). The color bar indicates the maximum and minimum SDM-Z values. Each study is labeled as a dot, with larger dots symbolizing larger sample sizes. Abbreviations: ReHo, regional homogeneity; MMSE, Mini-Mental State Examination; aMCl, amnestic mild cognitive impairment; DLPFC, dorsolateral prefrontal cortex; L, left; R, right; SDM, Seed-based d Mapping

has been proposed that a U-shaped curve of the medial temporal lobe activation takes place early in the course of prodromal AD [44]. However, to what extent of the compensation of local functional connectivity in the aging-aMCI-AD continuum, further longitudinal studies are warranted to calcify this question.

Recent studies have shown that aMCI is associated with an aberrant topological organization of large-scale functional brain networks beyond the DMN [41, 45, 46]. Apart from the regions within the DMN, our meta-analysis identified other regions of ReHo alterations in patients with aMCI, mainly involving the executive control network, visual network, and sensorimotor network. Regions of decreased ReHo in right DLPFC and superior parietal lobule are the components of the executive control network, which is implicated in initiating and modulating cognitive control. Despite the cardinal deficits in memory, patients with aMCI also exhibited impairments in executive functioning [47-50]. The decreased ReHo in the executive control network suggested the reduced executive control ability in patients with MCI. Additionally, our meta-analysis identified increased $\mathrm{ReHo}$ in the right lingual gyrus and left middle occipital gyrus. These regions are important nodes of the visual network, which is crucial for the visual information processing relating to visual cognition. Previous functional MRI studies suggested that the visual network in MCI was less affected than that in $\mathrm{AD}$ [41]. Impairment of visual cognition is pronounced in $\mathrm{AD}$ but not in aMCI $[51,52]$. Thus, increased ReHo in the visual network may be interpreted as a compensatory 


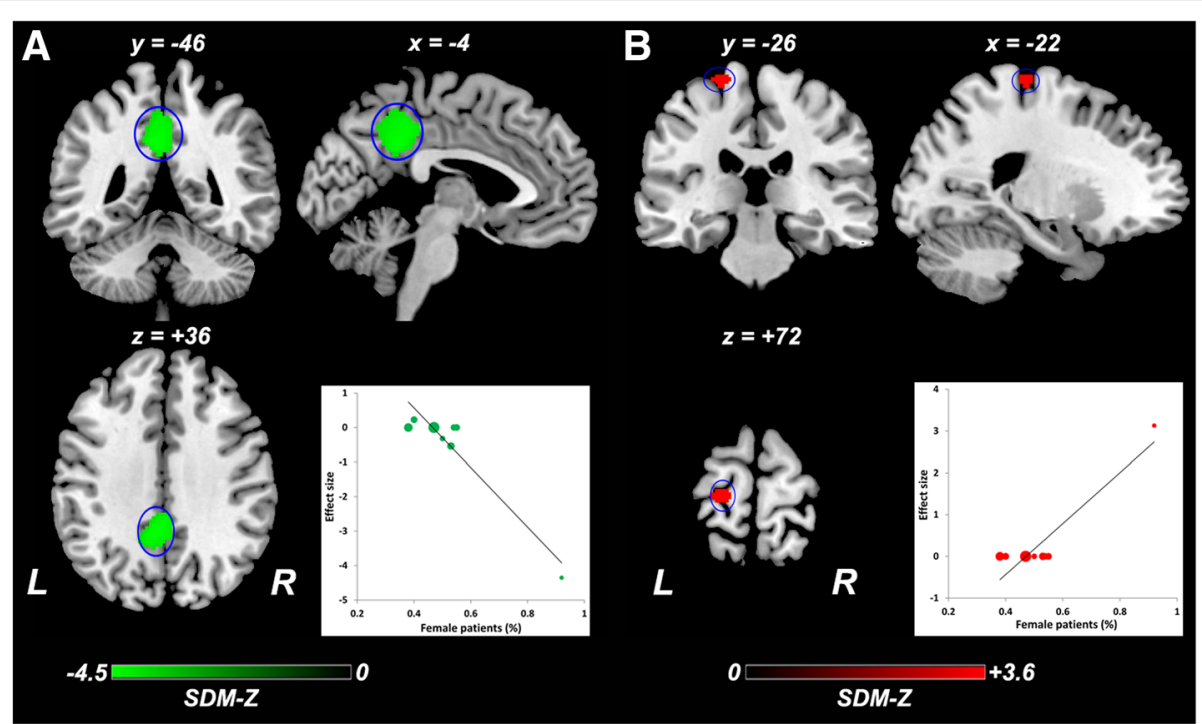

Fig. 5 Meta-regression analysis of ReHo alterations against the female ratio across studies. More females in the aMCl samples (female ratio) were related to lower ReHo in the left PCC/precuneus (a) and higher ReHo in the left pre/postcentral gyrus (b). The color bar indicates the maximum and minimum SDM-Z values. Each study is labeled as a dot, with larger dots symbolizing larger sample sizes. Abbreviations: ReHo, regional homogeneity; aMCl, amnestic mild cognitive impairment; L, left; R, right; SDM, Seed-based $d$ Mapping

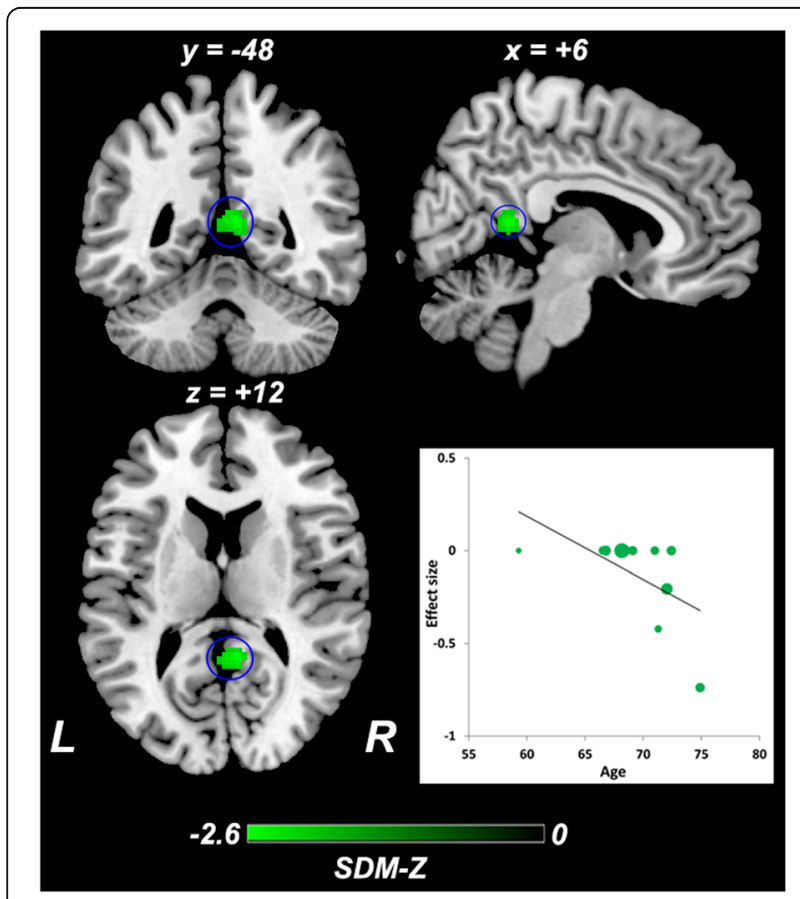

Fig. 6 Meta-regression analysis of ReHo alterations against the mean age across studies. Mean age in the aMCl sample was negatively correlated with ReHo in the right precuneus. The color bar indicates the maximum and minimum SDM-Z values. Each study is labeled as a dot, with larger dots symbolizing larger sample sizes. Abbreviations: ReHo, regional homogeneity; aMCl, amnestic mild cognitive impairment; $L$, left; $R$, right; SDM, Seed-based d Mapping process in patients with aMCI. We also observed consistent ReHo alterations within the sensorimotor network. Pathological or structural changes in the sensorimotor cortices were found to be least affected in aMCI and relatively preserved in AD [53-55]. Increased ReHo within the sensorimotor network might be compensated to disruptions of other functional networks.

Of note is that significant heterogeneity of ReHo alterations in the bilateral PCC, left parahippocampal gyrus/hippocampus, and right superior parietal lobule/ angular gyrus was observed in the meta-analysis. The subgroup meta-analyses indicated that MR field-strength and sex had confounding effects on the overall results of ReHo alterations. Exploratory meta-regression analyses revealed that this heterogeneity was attributed to some confounding factors, such as general cognitive function, gender distribution, age, and education level. Such moderators on brain structure or function have been well documented [39, 56-62]. Of interest, we observed significant association between ReHo alternates in the left $\mathrm{PCC} /$ precuneus and left pre/postcentral gyrus and female gender. Sex differences in brain ReHo [63], resting-state functional connectivity [64], and restingstate networks [65] in healthy subjects have been described. Although there are no statistically significant sex differences in the prevalence or incidence of aMCI [66]; however, epidemiologic studies indicate that women patients with $\mathrm{MCI}$ have greater longitudinal rates of cognitive and functional progression than men [67]. In postmortem investigations, women showed more extensive senile plaques deposition throughout the 


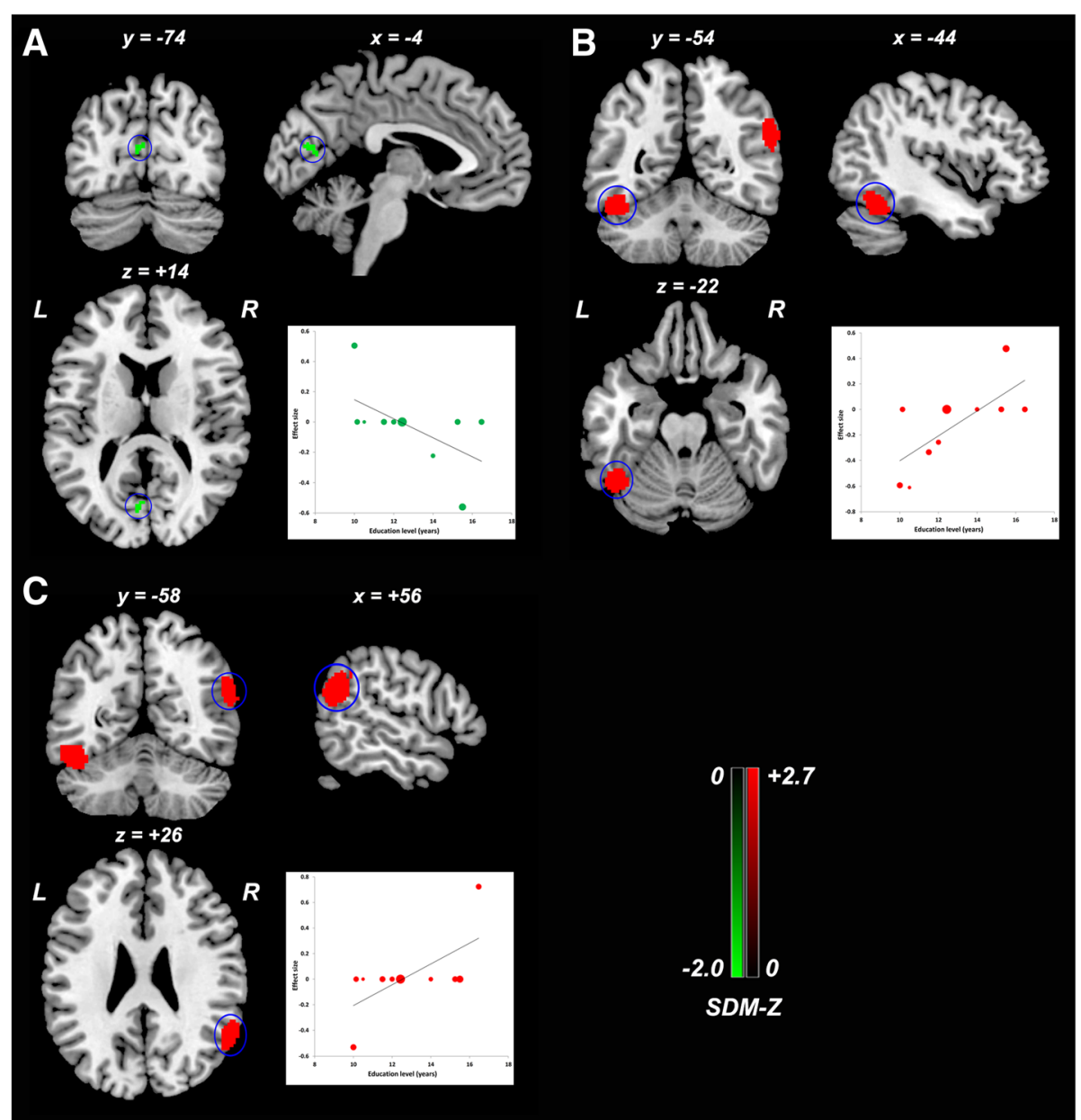

Fig. 7 Meta-regression analysis of ReHo alterations against the educational level across studies. Higher educational level in the aMCl sample was associated with lower ReHo in the left calcarine fissure/surrounding cortex (a), and higher ReHo in left fusiform gyrus/inferior temporal gyrus (b) and right angular gyrus/superior temporal gyrus (c). The color bar indicates the maximum and minimum SDM-Z values. Each study is labeled as a dot, with larger dots symbolizing larger sample sizes. Abbreviations: ReHo, regional homogeneity; aMCl, amnestic mild cognitive impairment; $L$, left; R, right; SDM, Seed-based d Mapping

brain than men at early neurofibrillary tangle stages [68]. Variability in brain ReHo in patients with aMCI due to sex differences is not well understood, which should be carefully studied in future studies. Although analyses of patient characteristics might help explain the heterogeneity observed across studies, they were far from sufficient. Other potential confounding factors, such as pathological heterogeneity underlying this disorder, genetic status, neuropsychological characteristics, administered medications, acquisition parameters of MRI, and preprocessing and analytic approaches, might also contribute to the heterogeneity. Unfortunately, it was not possible to conduct separate subgroup and meta-regression analyses to systemically examine these moderating effects because of insufficient information available from the original studies, but these confounding effects merit future investigations.
Several limitations in the present study should be noted. First, we observed some publication biases in the bilateral PCC and left parahippocampal gyrus/hippocampus. In order to increase the quality of the meta-analysis, only peer-reviewed articles using a whole-brain analysis published in English were included in this meta-analysis. Thus, studies published in other languages, unpublished materials, or studies performing with ROI and two-dimensional analyses were excluded. In addition, of the 11 datasets included in the meta-analysis, eight were from China (280 patients with aMCI), two from the Alzheimer's Disease Neuroimaging Initiative (ADNI) database (64 patients with aMCI), and one from Korea (34 patients with aMCI). Due to these factors, publication bias might be not avoidable. Hence, an additional research need exists for future comprehensively pooling big neuroimaging data from worldwide populations. 
Table 4 Meta-regression analyses

\begin{tabular}{|c|c|c|c|c|}
\hline Anatomical label & Peak MNI coordinate $(x, y, z)$ & Voxels & SDM-Z value & $p$ value \\
\hline \multicolumn{5}{|l|}{ Effect of general cognitive function measured with MMSE score } \\
\hline A. Right angular gyrus/supramarginal gyrus (BAs 39, 40, and 22) & $58,-50,24$ & 371 & -2.3 & 0.0001 \\
\hline B. Right dorsolateral prefrontal cortex (BA 9) & $38,14,34$ & 139 & -2.8 & 0.00004 \\
\hline C. Left inferior temporal gyrus (BA 20) & $-52,-52,-14$ & 97 & -2.2 & 0.0002 \\
\hline \multicolumn{5}{|l|}{ Effect of gender distribution } \\
\hline A. Left posterior cingulate gyrus/precuneus (BA 23) & $-4,-46,36$ & 689 & -4.5 & 0.00002 \\
\hline B. Left pre/postcentral gyrus (BAs 6 and 4) & $-22,-26,72$ & 50 & 3.6 & 0.0002 \\
\hline \multicolumn{5}{|l|}{ Effect of age } \\
\hline Right precuneus (BA 29) & $6,-48,12$ & 121 & -2.6 & 0.00004 \\
\hline \multicolumn{5}{|l|}{ Effect of education level } \\
\hline A. Left calcarine fissure/surrounding cortex (BAs 17 and 18) & $-4,-74,14$ & 25 & -2.0 & 0.0002 \\
\hline B. Left fusiform gyrus/inferior temporal gyrus (BAs 37 and 20) & $-44,-54,-22$ & 321 & 2.7 & 0.00003 \\
\hline C. Right angular gyrus/superior temporal gyrus (BAs 39, 40 and 22) & $56,-58,26$ & 315 & 2.6 & 0.00004 \\
\hline
\end{tabular}

Abbreviations: MMSE Mini-Mental State Examination, MNI Montreal Neurological Institute, SDM Seed-based $d$ Mapping, BA Brodmann Area

Second, although patients with aMCI are heterogeneous in etiology, the ReHo findings in our meta-analysis may represent a common pattern of functional changes underlying aMCI. However, limited by the cross-sectional design of the original studies included, further clinical outcomes of patients with aMCI that convert to $\mathrm{AD}$ or remain stable or improve can vary considerably. Our study could not disentangle the ReHo differences between them. In addition, the cross-sectional design of the studies could not elucidate the causality of the alterations in ReHo and aMCI. Further longitudinal follow-up designed studies are highly warranted to clarify these issues. Third, as already mentioned above, many confounding factors might influence ReHo alterations on aMCI. However, not all studies made enough adjustment for these confounders. To reduce heterogeneity and provide more conclusive findings, larger and better designed studies in homogenous patients are needed. Finally, the coordinate-based metaanalysis approaches are important for identifying the anatomical convergence of neuroimaging studies. However, they mainly relied on published coordinates and their effect sizes, not on original imaging data, which may bias the results.

\section{Conclusions}

This voxel-wise meta-analysis provides provisional evidence that aMCI is associated with abnormal ReHo within the DMN, executive control network, visual network, and sensorimotor network. The decreased and increased local connectivity in aMCI suggest coexistence of functional deficits and compensation in these networks. These findings contribute to the modeling of brain functional connectomes and to a better understanding of the neural substrates underlying cognitive deficits associated with aMCI. This meta-analysis also provides quantitative evidence that general cognitive function, gender distribution, age, and education level are confounding factors that lead to heterogeneity in the results and merit future investigations.

\section{Additional files}

Additional file 1: Criteria for objective assessment of methodological quality of individual studies. Notes: A maximum score of 20 for each study, allocated as per the criteria specified above. (DOCX $19 \mathrm{~kb}$ )

Additional file 2: Subgroup meta-analysis of studies using 3.0 T MRI scanners ( $N=9$ ). Abbreviations: $N$, number of datasets; ReHo, Regional Homogeneity; MNI, Montreal Neurological Institute; SDM, Seed-based d Mapping; BA, Brodmann area. (DOCX $25 \mathrm{~kb}$ )

Additional file $\mathbf{3}$ Subgroup meta-analysis of studies that matched for sex $(N=10)$. Abbreviations: $N$, number of datasets; ReHo, Regional Homogeneity; MNI, Montreal Neurological Institute; SDM, Seed-based d Mapping; BA, Brodmann area. (DOCX 24 kb)

\section{Abbreviations}

AD: Alzheimer's dementia; ADNI: Alzheimer's Disease Neuroimaging Initiative; AFNI: Analysis of Functional Neurolmages; aMCl: Amnestic mild cognitive impairment; BA: Brodmann area; BRAT: Brainnetome fMRI toolkit; Cl: Confidence interval; DLPFC: Dorsolateral prefrontal cortex; DMN: Default mode network; DPARSF: Data Processing Assistant for Resting-State fMRI software; DPARSFA: DAPRSF Advanced edition; FWHM: Full-width at half maximum; HC: Healthy control; MMSE: Mini-Mental State Examination; MNI: Montreal Neurological Institute; MOOSE: Meta-analysis Of Observational Studies in Epidemiology; NA: Not Available; PCC: Posterior cingulate cortex; ReHo: Regional homogeneity; REST: The Resting-State fMRI Data Analysis Toolkit; ROI: Region of interest; rs-fMRI: Resting-state functional magnetic resonance imaging; SD: Standard Deviation; SDM: Seed-based d Mapping; SMD: Standardized mean difference; SPM: Statistical Parametric Mapping

\section{Acknowledgements}

We thank all the authors of the included studies. 


\section{Funding}

This work was supported by the National Natural Science Foundation of China (Grant No. 81601161) and Jiangsu Provincial Commission of Health and Family Planning (Grant No. QNRC2016466).

\section{Availability of data and materials}

The datasets supporting the conclusions of this article are included in the article and its additional files.

\section{Authors' contributions}

PLP, ZYD, DZ, and WX design the whole study. DZ, WX, ZQY, and PWZ searched and selected the studies, analyzed the data, and prepared figures. PLP undertook the statistical analysis. ZYD, PLP, HCS, HLL, and JGZ participated in the interpretation of data. DZ and WX wrote the manuscript. PLP and ZYD revised the manuscript. All authors read and approved the final manuscript.

\section{Ethics approval and consent to participate}

This article does not contain any studies with human participants performed by any of the authors.

\section{Consent for publication}

$$
\text { Not applicable. }
$$

\section{Competing interests}

The authors declare that they have no competing interests.

\section{Author details}

'School of Nursing, Jiangsu Vocational College of Medicine, Yancheng, People's Republic of China. ${ }^{2}$ Department of Neurology, Affiliated Yancheng Hospital, School of Medicine, Southeast University, West Xindu Road 2\#, Yancheng, Jiangsu Province 224001, People's Republic of China. ${ }^{3}$ Department of Central Laboratory, Affiliated Yancheng Hospital, School of Medicine, Southeast University, West Xindu Road 2\#, Yancheng, Jiangsu Province 224001, People's Republic of China. ${ }^{4}$ Department of Radiology, Affiliated Yancheng Hospital, School of Medicine, Southeast University, West Xindu Road 2\#, Yancheng, Jiangsu Province 224001, People's Republic of China.

\section{Received: 15 May 2018 Accepted: 11 October 2018}

\section{Published online: 07 November 2018}

\section{References}

1. Petersen RC. Mild cognitive impairment as a diagnostic entity. J Intern Med. 2004:256:183-94.

2. Mitchell AJ, Shiri-Feshki M. Rate of progression of mild cognitive impairment to dementia--meta-analysis of 41 robust inception cohort studies. Acta Psychiatr Scand. 2009;119:252-65.

3. Petersen RC, Roberts RO, Knopman DS, Boeve BF, Geda YE, Ivnik RJ, et al. Mild cognitive impairment: ten years later. Arch Neurol. 2009;66:1447-55.

4. Barkhof $F$, Haller S, Rombouts SA. Resting-state functional MR imaging: a new window to the brain. Radiology. 2014;272:29-49.

5. Smitha KA, Akhil Raja K, Arun KM, Rajesh PG, Thomas B, Kapilamoorthy TR, et al. Resting state fMRI: a review on methods in resting state connectivity analysis and resting state networks. Neuroradiol J. 2017;30:305-17.

6. Liu Y, Wang K, Yu C, He Y, Zhou Y, Liang M, et al. Regional homogeneity, functional connectivity and imaging markers of Alzheimer's disease: a review of resting-state fMRI studies. Neuropsychologia. 2008;46:1648-56.

7. Rektorova I. Resting-state networks in Alzheimer's disease and Parkinson's disease. Neurodegener Dis. 2014:13:186-8.

8. Jiang L, Zuo XN. Regional homogeneity: a multimodal, multiscale neuroimaging marker of the human connectome. Neuroscientist. 2016;22:486-505

9. Zang $Y$, Jiang T, Lu Y, He Y, Tian L. Regional homogeneity approach to fMRI data analysis. Neurolmage. 2004;22:394-400.

10. Long $Z$, Jing B, Yan $H$, Dong J, Liu $H$, Mo X, et al. A support vector machinebased method to identify mild cognitive impairment with multi-level characteristics of magnetic resonance imaging. Neuroscience. 2016:331:169-76

11. Zhang Z, Liu Y, Jiang T, Zhou B, An N, Dai H, et al. Altered spontaneous activity in Alzheimer's disease and mild cognitive impairment revealed by regional homogeneity. Neurolmage. 2012;59:1429-40.
12. Zhang $Z$, Deng $L$, Bai $F$, Shi $Y, Y u H$, Yuan $Y$, et al. Alteration of resting brain function by genetic variation in angiotensin converting enzyme in amnestic-type mild cognitive impairment of Chinese Han. Behav Brain Res. 2010;208:619-25.

13. Yuan X, Han Y, Wei Y, Xia M, Sheng C, Jia J, et al. Regional homogeneity changes in amnestic mild cognitive impairment patients. Neurosci Lett. 2016;629:1-8.

14. Ni L, Liu R, Yin Z, Zhao H, Nedelska Z, Hort J, et al. Aberrant spontaneous brain activity in patients with mild cognitive impairment and concomitant lacunar infarction: a resting-state functional MRI study. J Alzheimers Dis. 2016:50:1243-54.

15. Norman L, Carlisi C, Lukito S, Hart H, Mataix-Cols D, Radua J, et al. Structural and functional brain abnormalities in attention-deficit/hyperactivity disorder and obsessive-compulsive disorder: a comparative meta-analysis. JAMA Psychiatry. 2016;73:815-25.

16. Luijten M, Schellekens AF, Kuhn S, Machielse MW, Sescousse G. Disruption of reward processing in addiction : an image-based meta-analysis of functional magnetic resonance imaging studies. JAMA Psychiatry. 2017:74:387-98

17. Ma HR, Sheng LQ, Pan PL, Wang GD, Luo R, Shi HC, et al. Cerebral glucose metabolic prediction from amnestic mild cognitive impairment to Alzheimer's dementia: a meta-analysis. Translat Neurodegeneration. 2018;7:9.

18. Stroup DF, Berlin JA, Morton SC, Olkin I, Williamson GD, Rennie D, et al. Meta-analysis of observational studies in epidemiology: a proposal for reporting. Meta-analysis of observational studies in epidemiology (MOOSE) group. JAMA. 2000;283:2008-12.

19. Radua J, Grau M, van den Heuvel OA, Thiebaut de Schotten M, Stein DJ, Canales-Rodriguez EJ, et al. Multimodal voxel-based meta-analysis of white matter abnormalities in obsessive-compulsive disorder. Neuropsychopharmacology. 2014;39:1547-57.

20. Radua J, Mataix-Cols D. Voxel-wise meta-analysis of grey matter changes in obsessive-compulsive disorder. Br J Psychiatry. 2009;195:393-402.

21. Radua J, Mataix-Cols D, Phillips ML, El-Hage W, Kronhaus DM, Cardoner N, et al. A new meta-analytic method for neuroimaging studies that combines reported peak coordinates and statistical parametric maps. Eur Psychiatry. 2012;27:605-11.

22. Lim L, Radua J, Rubia K. Gray matter abnormalities in childhood maltreatment: a voxel-wise meta-analysis. Am J Psychiatry. 2014;171:854-63.

23. Alegria AA, Radua J, Rubia K. Meta-analysis of $\mathrm{fMRI}$ studies of disruptive behavior disorders. Am J Psychiatry. 2016;173:1119-30.

24. Pan P, Zhan H, Xia M, Zhang Y, Guan D. Xu Y. aberrant regional homogeneity in Parkinson's disease: a voxel-wise meta-analysis of restingstate functional magnetic resonance imaging studies. Neurosci Biobehav Rev. 2017:72:223-31

25. Wise T, Radua J, Nortje G, Cleare AJ, Young AH, Arnone D. Voxel-based meta-analytical evidence of structural Disconnectivity in major depression and bipolar disorder. Biol Psychiatry. 2016;79:293-302.

26. Li W, Chen Z, Wu M, Zhu H, Gu L, Zhao Y, et al. Characterization of brain blood flow and the amplitude of low-frequency fluctuations in major depressive disorder: a multimodal meta-analysis. J Affect Disord. 2016;210:303-11.

27. Liu Z, Wei W, Bai L, Dai R, You Y, Chen S, et al. Exploring the patterns of acupuncture on mild cognitive impairment patients using regional homogeneity. PLoS One. 2014;9:e99335.

28. Luo X, Jiaerken $Y$, Huang $P, X u X J$, Qiu T, Jia Y, et al. Alteration of regional homogeneity and white matter hyperintensities in amnestic mild cognitive impairment subtypes are related to cognition and CSF biomarkers. Brain Imaging Behav. 2018;12:188-200.

29. Wang $Y$, Zhao X, Xu S, Yu L, Wang L, Song M, et al. Using regional homogeneity to reveal altered spontaneous activity in patients with mild cognitive impairment. Biomed Res Int. 2015;2015:807093.

30. Yuan B, Chen J, Gong L, Shu H, Liao W, Wang Z, et al. Mediation of episodic memory performance by the executive function network in patients with amnestic mild cognitive impairment: a resting-state functional MRI study. Oncotarget. 2016;7:64711-25

31. Bai F, Zhang Z, Yu H, Shi Y, Yuan Y, Zhu W, et al. Default-mode network activity distinguishes amnestic type mild cognitive impairment from healthy aging: a combined structural and resting-state functional MRI study. Neurosci Lett. 2008:438:111-5.

32. Cha J, Hwang JM, Jo HJ, Seo SW, Na DL, Lee JM. Assessment of functional characteristics of amnestic mild cognitive impairment and Alzheimer's 
disease using various methods of resting-state FMRI analysis. Biomed Res Int. 2015;2015:907464.

33. Pievani M, Pini L, Ferrari C, Pizzini FB, Boscolo Galazzo I, Cobelli C, et al. Coordinate-based meta-analysis of the default mode and salience network for target identification in non-invasive brain stimulation of Alzheimer's disease and behavioral variant frontotemporal dementia networks. J Alzheimers Dis. 2017;57:825-43.

34. Wang C, Pan Y, Liu Y, Xu K, Hao L, Huang F, et al. Aberrant default mode network in amnestic mild cognitive impairment: a meta-analysis of independent component analysis studies. Neurol Sci. 2018;39(5):919-31.

35. Wang L, Li H, Liang Y, Zhang J, Li X, Shu N, et al. Amnestic mild cognitive impairment: topological reorganization of the default-mode network. Radiology. 2013;268:501-14.

36. Petrella JR, Sheldon FC, Prince SE, Calhoun VD, Doraiswamy PM. Default mode network connectivity in stable vs progressive mild cognitive impairment. Neurology. 2011;76:511-7.

37. Serra L, Cercignani M, Mastropasqua C, Torso M, Spano B, Makovac E, et al. Longitudinal changes in functional brain connectivity predicts conversion to Alzheimer's disease. J Alzheimers Dis. 2016;51:377-89.

38. Qi Z, Wu X, Wang Z, Zhang N, Dong H, Yao L, et al. Impairment and compensation coexist in amnestic MCl default mode network. Neurolmage. 2010;50:48-55

39. Pan P, Zhu L, Yu T, Shi H, Zhang B, Qin R, et al. Aberrant spontaneous lowfrequency brain activity in amnestic mild cognitive impairment: a metaanalysis of resting-state fMRI studies. Ageing Res Rev. 2017;35:12-21.

40. Jacobs HI, Radua J, Luckmann HC, Sack AT. Meta-analysis of functional network alterations in Alzheimer's disease: toward a network biomarker. Neurosci Biobehav Rev. 2013;37:753-65.

41. Li HJ, Hou XH, Liu HH, Yue CL, He Y, Zuo XN. Toward systems neuroscience in mild cognitive impairment and Alzheimer's disease: a meta-analysis of 75 fMRI studies. Hum Brain Mapp. 2015;36:1217-32.

42. Liang P, Li Z, Deshpande G, Wang Z, Hu X, Li K. Altered causal connectivity of resting state brain networks in amnesic MCI. PLoS One. 2014;9:e88476.

43. Lau WK, Leung MK, Lee TM, Law AC. Resting-state abnormalities in amnestic mild cognitive impairment: a meta-analysis. Transl Psychiatry. 2016;6:e790.

44. Dickerson BC, Sperling RA. Large-scale functional brain network abnormalities in Alzheimer's disease: insights from functional neuroimaging. Behav Neurol. 2009;21:63-75.

45. Cai S, Chong T, Peng Y, Shen W, Li J, von Deneen KM, et al. Altered functional brain networks in amnestic mild cognitive impairment: a resting-state fMRI study. Brain Imaging Behav. 2017;11:619-31.

46. Dai Z, He Y. Disrupted structural and functional brain connectomes in mild cognitive impairment and Alzheimer's disease. Neurosci Bull. 2014;30:217-32.

47. Traykov L, Raoux N, Latour F, Gallo L, Hanon O, Baudic S, et al. Executive functions deficit in mild cognitive impairment. Cogn Behav Neurol. 2007:20:219-24

48. Aretouli E, Brandt J. Everyday functioning in mild cognitive impairment and its relationship with executive cognition. Int I Geriatr Psychiatry. 2010;25:224-33.

49. Saunders NL, Summers MJ. Longitudinal deficits to attention, executive, and working memory in subtypes of mild cognitive impairment. Neuropsychology. 2011;25:237-48.

50. Brandt J, Aretouli E, Neijstrom E, Samek J, Manning K, Albert MS, et al. Selectivity of executive function deficits in mild cognitive impairment. Neuropsychology. 2009;23:607-18.

51. Uhlhaas PJ, Pantel J, Lanfermann H, Prvulovic D, Haenschel C, Maurer K, et al. Visual perceptual organization deficits in Alzheimer's dementia. Dement Geriatr Cogn Disord. 2008;25:465-75.

52. Mandal PK, Joshi J, Saharan S. Visuospatial perception: an emerging biomarker for Alzheimer's disease. J Alzheimers Dis. 2012;31 (Suppl 3):S117-35.

53. Frisoni $G B$, Prestia $A$, Rasser $P E$, Bonetti $M$, Thompson PM. In vivo mapping of incremental cortical atrophy from incipient to overt Alzheimer's disease. J Neurol. 2009;256:916-24.

54. Pearson RC, Esiri MM, Hiorns RW, Wilcock GK, Powell TP. Anatomical correlates of the distribution of the pathological changes in the neocortex in Alzheimer disease. Proc Natl Acad Sci U S A. 1985;82:4531-4.

55. Karas GB, Burton EJ, Rombouts $S A$, van Schijndel RA, O'Brien JT, Scheltens $P$, et al. A comprehensive study of gray matter loss in patients with Alzheimer's disease using optimized voxel-based morphometry. Neurolmage. 2003;18:895-907.
56. Li HJ, Hou XH, Liu HH, Yue CL, Lu GM, Zuo XN. Putting age-related task activation into large-scale brain networks: a meta-analysis of 114 fMRI studies on healthy aging. Neurosci Biobehav Rev. 2015;57:156-74.

57. Sullivan EV, Rosenbloom M, Serventi KL, Pfefferbaum A. Effects of age and sex on volumes of the thalamus, pons, and cortex. Neurobiol Aging. 2004;25:185-92.

58. Di X, Rypma B, Biswal BB. Correspondence of executive function related functional and anatomical alterations in aging brain. Prog NeuroPsychopharmacol Biol Psychiatry. 2014;48:41-50.

59. Ruigrok AN, Salimi-Khorshidi G, Lai MC, Baron-Cohen S, Lombardo MV, Tait $\mathrm{RJ}$, et al. A meta-analysis of sex differences in human brain structure. Neurosci Biobehav Rev. 2014;39:34-50.

60. Franzmeier N, Buerger K, Teipel S, Stern Y, Dichgans M, Ewers M. Cognitive reserve moderates the association between functional network anticorrelations and memory in MCl. Neurobiol Aging. 2017;50:152-62.

61. Nickl-Jockschat T, Kleiman A, Schulz JB, Schneider F, Laird AR, Fox PT, et al. Neuroanatomic changes and their association with cognitive decline in mild cognitive impairment: a meta-analysis. Brain Struct Funct. 2012;217:115-25.

62. Skup M, Zhu H, Wang Y, Giovanello KS, Lin JA, Shen D, et al. Sex differences in grey matter atrophy patterns among $A D$ and aMCl patients: results from ADNI. Neurolmage. 2011;56:890-906

63. Xu C, Li C, Wu H, Wu Y, Hu S, Zhu Y, et al. Gender differences in cerebral regional homogeneity of adult healthy volunteers: a resting-state FMRI study. Biomed Res Int. 2015;2015:183074.

64. Filippi M, Valsasina P, Misci P, Falini A, Comi G, Rocca MA. The organization of intrinsic brain activity differs between genders: a resting-state fMRI study in a large cohort of young healthy subjects. Hum Brain Mapp. 2013;34:1330-43.

65. Scheinost D, Finn ES, Tokoglu F, Shen X, Papademetris X, Hampson M, et al. Sex differences in normal age trajectories of functional brain networks. Hum Brain Mapp. 2015;36:1524-35.

66. Au B. Dale-McGrath S, Tierney MC. Sex differences in the prevalence and incidence of mild cognitive impairment: a meta-analysis. Ageing Res Rev. 2017;35:176-99.

67. Lin KA, Choudhury KR, Rathakrishnan BG, Marks DM, Petrella JR, Doraiswamy PM. Marked gender differences in progression of mild cognitive impairment over 8 years. Alzheimers Dement. 2015;1:103-10.

68. Corder EH, Ghebremedhin E, Taylor MG, Thal DR, Ohm TG, Braak H. The biphasic relationship between regional brain senile plaque and neurofibrillary tangle distributions: modification by age, sex, and APOE polymorphism. Ann N Y Acad Sci. 2004;1019:24-8.

\section{Ready to submit your research? Choose BMC and benefit from:}

- fast, convenient online submission

- thorough peer review by experienced researchers in your field

- rapid publication on acceptance

- support for research data, including large and complex data types

- gold Open Access which fosters wider collaboration and increased citations

- maximum visibility for your research: over $100 \mathrm{M}$ website views per year

At $\mathrm{BMC}$, research is always in progress.

Learn more biomedcentral.com/submissions 